\title{
Intervertebral Disc Segmentation and Volumetric Reconstruction from Peripheral Quantitative Computed Tomography Imaging
}

\author{
Alexander Wong, Akshaya Mishra, Justin Yates, Paul Fieguth, David A. Clausi, and Jack P. Callaghan
}

\begin{abstract}
An automatic system for segmenting and constructing volumetric representations of intervertebral discs from Peripheral Quantitative Computed Tomography (PQCT) imagery is presented. The system is designed to allow for automatic quantitative analysis of progressive herniation damage to the intervertebral discs under flexion/extension motions combined with a compressive load. Automatic segmentation and volumetric reconstruction of intervertebral disc from $P Q C T$ imagery is a very challenging problem due to factors such as streak artifacts and unclear material density separation between contrasted intervertebral disc and surrounding bone in the PQCT imagery, as well as the formation of multiple contrasted regions under axial scans. To address these factors, a novel multi-scale level set approach based on the Mumford Shah energy functional in iterative bilateral scale space is employed to segment the intervertebral disc regions from the PQCT imagery. A Delaunay triangulation is then performed based on the set of points associated with the intervertebral disc regions to construct the volumetric representation of the intervertebral disc. Experimental results show that the proposed system achieves accurate segmentation and volumetric reconstructions of intervertebral discs when compared to ground-truth measurements.
\end{abstract}

Index Terms-computed tomography, intervertebral disc, scale space, Mumford Shah, segmentation, volumetric reconstruction.

\section{INTRODUCTION}

One of the most common musculoskeletal disorders faced in industrialized countries is low back pain [1]. While the factors that contribute to low back pain can vary, a frequent source of low back pain is damage to the intervertebral discs, which act as shock absorbers between the individual vertebrae. In particular, studies have shown that the major factors contributing to intervertebral disc damage are flexion/compressive motions [2] and compressive load [3]. Hence, to improve the understanding of intervertebral disc damage, it is necessary to study and quantify the migration of nucleus pulposus in herniated intervertebral discs under flexion/compressive motions combined with a compressive load.

In established non-destructive approaches, progressive intervertebral disc herniation damage was studied using standard $\mathrm{x}$-ray radiographic imagery. However, comparative studies have demonstrated that such approaches show discordance

\footnotetext{
Alexander Wong, Akshaya Mishra, Paul Fieguth, and David A. Clausi are with Dept. of Systems Design Engineering, U. of Waterloo, Waterloo, Canada N2L 3G1. \{a28wong, akmishra, pfieguth, dclausi\}@uwaterloo.ca Justin Yates and Jack P. Callaghan are with Dept. of Kinesiology, U. of Waterloo, Waterloo, Canada N2L 3G1. jyates@uwaterloo.ca, callagha@healthy.uwaterloo.ca
}

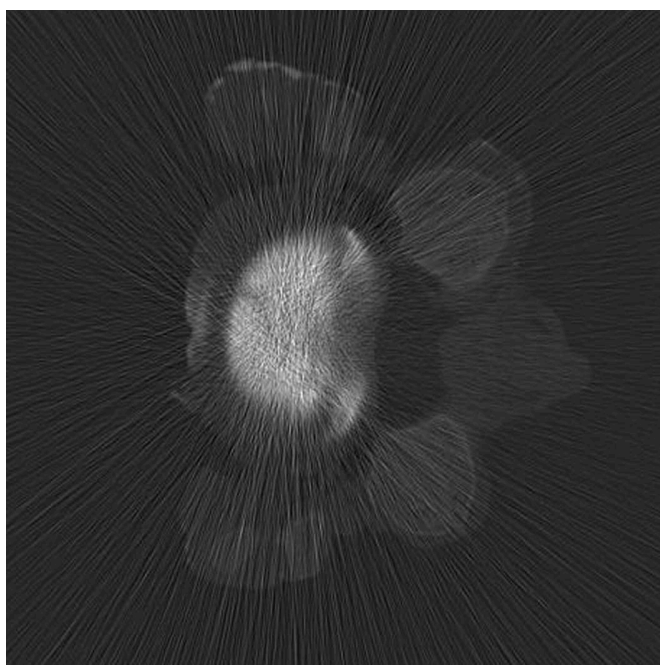

Fig. 1. An axial CT image slice of the intervertebral disc and surrounding vertebral regions. The intervertebral disc is represented by the bright regions due to the injected contrast agent. Noticeable streak artifacts and unclear material density separation between contrast agent and surrounding bone in the PQCT imagery, along with multiple contrasted regions make segmenting the intervertebral disc in an automated manner a difficult challenge.

with destructive 'gold standard' dissection techniques [4], [5]. A promising new non-destructive approach for studying intervertebral disc damage is the use of computed tomography (CT). To study progressive intervertebral disc herniation, a contrast agent is injected into the intervertebral disc prior to being imaged using CT. A typical axial CT image slice of the intervertebral disc and surrounding vertebral regions is shown in Fig. 1, where the intervertebral disc is represented by the bright regions due to the injected contrast agent. One of the advantages of CT imagery over traditional x-ray radiographic imagery is that $\mathrm{CT}$ provides improved resolution and material density sensitivity, and as such holds the potential to better illustrate actual disc damage previously observed using dissection techniques without damaging the specimen. Therefore, a model of herniation damage constructed from CT imagery may allow a better visualization of herniation damage without specimen destruction, and holds the potential to add valuable information regarding herniation progression and specific pathways of damage.

The visualization and quantitative analysis of progressive intervertebral disc herniation damage requires the automatic segmentation and volumetric reconstruction of intervertebral discs from the acquired CT imagery, which is a very chal- 
lenging problem for several reasons. First, a basic assumption made in CT imaging is that some transmitted radiation is observed by each detector at every position [6]. However, the presence of a high density material can significantly reduce transmission to an extent that a detector observes no transmission. The high density material in the case of studying intervertebral disc herniation using CT imagery is the contrast agent injected into the intervertebral disc. This violation in the basic assumption of CT imaging results in streaking artifacts in the reconstructed image, which is very noticeable in Fig. 1. Second, the material density separation between the contrasted intervertebral disc regions and the surrounding bone regions is unclear and appears blurred in the CT image, making it difficult to isolate the intervertebral disc regions. Third, multiple contrasted regions are formed under axial CT scans, which makes it necessary to segment multiple regions from the image. The proposed method aims to address these issues to provide accurate segmentation and volumetric reconstruction of intervertebral discs.

The main contribution of this paper is an automated system for segmentation and volumetric reconstruction of intervertebral disc from Peripheral Quantitative Computed Tomography (PQCT) imagery. The proposed method is highly robust to streaking artifacts and unclear material density separation between contrasted intervertebral and surrounding bone regions, which are the key challenges to intervertebral disc segmentation in CT imagery. To the best of the authors' knowledge, there is no research literature associated with automatic segmentation and volumetric reconstruction of intervertebral disc. In this paper, the materials and methods are described in Section II, and the experimental results using PQCT images of porcine spinal functional spinal units are presented in Section III.

\section{Materials AND Methods}

The materials and methods used to study progressive intervertebral disc herniation under flexion/extension motions combined with a compressive load can be described as follows. A solution consisting of $0.4 \mathrm{ml}$ of radio-opaque contrast agent (Omnipaque $^{\mathrm{TM}}$, General Electric Company, USA) and 0.15 $\mathrm{ml}$ of blue dye was injected into the intervertebral discs of 64 porcine functional spinal units. A photograph of a porcine functional spinal unit with the contrast agent injected into the intervertebral disc is shown in Fig. 2. Damage in the form of intervertebral herniations was created by loading the specimens in a servo-hydraulic testing system under combined compressive loading with repeated flexion/extension motions [7]. Three sets of CT images were then obtained; prior to loading, after sufficient cycles of load to cause partial damage to the intervertebral disc, and again after additional loading hypothesized to exacerbate herniation damage. The CT images were taken through the level of the intervertebral disc using a Stratec Medizntechnik XCT 2000 Research Plus Peripheral Quantitative Computed Tomography (PQCT) scanner. Each of the three sets of CT images consists of eight serial slices spanning the entire intervertebral disc using a slice thickness of $0.9 \mathrm{~mm}$, a scan speed of $10 \mathrm{~mm} / \mathrm{s}$ and implementing a voxel

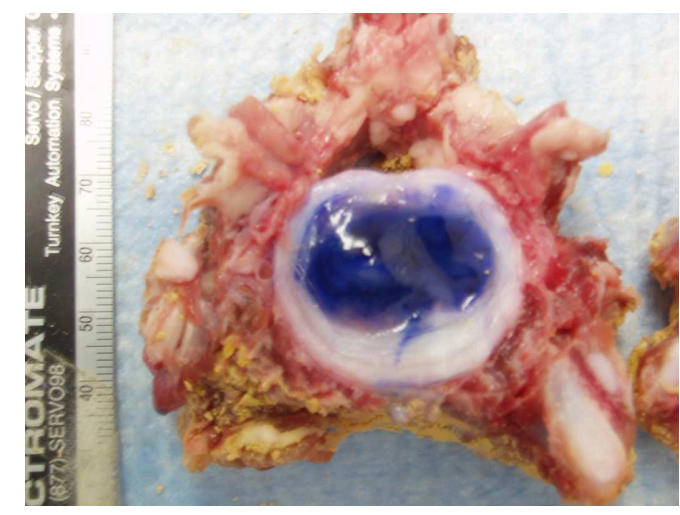

Fig. 2. A photograph of a porcine functional spinal unit with the contrast agent (shown in blue due to a blue dye mixed in with the contrast agent) injected into the intervertebral disc.

size of $0.2 \mathrm{~mm} \times 0.2 \mathrm{~mm} \times 0.2 \mathrm{~mm}$. For each set of CT images, the contrasted intervertebral disc regions are then segmented from the surrounding bone structures using a multi-scale level set approach based on the Mumford Shah energy functional in iterative bilateral scale space. A volumetric reconstruction of intervertebral disc is then constructed from the segmented intervertebral disc regions using Delaunay triangulation [8].

\section{A. Multi-scale Segmentation in Iterative Bilateral Scale Space}

The first step in the proposed system is to segment the contrasted intervertebral disc regions from the PQCT imagery. One of the most common methods for image segmentation is based on the minimization of the Mumford-Shah energy functional [9]. Let the image $u_{0}$ be a piece-wise smooth function that is well approximated by a set of smooth functions $u_{i}$ defined on a set of regions $R_{i}$ covering plane domain $R$. In our case, $u_{0}$ is a function of material density and the set of regions $R_{i}$ is defined as the contrasted invertebral disc and surrounding bone regions. The segmentation problem can then be defined as determining the decomposition $R=R_{1} \bigcup \ldots \bigcup R_{n}$ of $u_{0}$ that provides the optimal estimate of $u_{0}$ by a set of smooth functions $u_{1}, \ldots, u_{n}$. The decomposition of $u_{0}$ can be obtained by minimizing the Mumford-Shah energy functional, which indicates the degree of match between $u_{0}$ and the estimated decomposition,

$$
\hat{R}=\underset{R}{\arg \min }\left[\alpha \oint_{R}\left(u-u_{0}\right)^{2} d \underline{x}+\oint_{R-\Gamma}\|\nabla u\|^{2} d \underline{x}+\beta|\Gamma|\right],
$$

where $\Gamma$ is the boundary between regions, $|\Gamma|$ is the total arclength of $\Gamma$, and $\alpha$ and $\beta$ controls the penalty terms.

There are three main issues that need to be dealt with to provide accurate intervertebral segmentation results using the Mumford-Shah energy minimization approach. First, due to the shape of the intervertebral disc, particularly after herniation, axial CT scans of the intervertebral disc can result in multiple contrasted regions that need to be segmented. Second, the density separation between the contrasted regions and surround bone regions become unclear near the boundaries of 
the regions, making it difficult to establish a clear boundary between the regions. Third, significant streak artifacts exhibited in the PQCT imagery can result in poor segmentation as well as slow convergence. An effective approach for addressing the issue associated with multiple contrasted regions is to use the level-set method [10] for minimizing the Mumford-Shah energy functional. One of the main advantages of using the level-set method is that it automatically handles topological changes, thus making it well suited for segmenting multiple contrasted intervertebral disc regions within the same CT image. To minimize the Mumford-Shah energy functional using the level-set method, $\Gamma$ is treated as a zero level set function $\phi_{0}$ of a surface $\phi$ and evolved based on a partial differential equation towards the minimum energy potential.

To address the issues associated with streaking artifacts and unclear region separation, we propose a multi-scale extension to solving the Mumford-Shah energy minimization problem in an iterative bilateral scale space. The concept of iterative bilateral scale space is inspired by the bilateral filtering scheme proposed by Tomasi and Manduchi [11], where detail in an image is smoothed based on not only spatial locality but also photometric differences. We take the fundamental concept behind bilateral filtering and extend it based on scale space theory [12], where a multi-scale decomposition of an image is formed such that image detail is monotonically removed at each subsequent scale. For a given image $u_{0}$, the corresponding iterative bilateral scale-space representation is defined by a family of derived images $L_{t}$, where,

$$
L_{t}(\underline{x})=\frac{\sum_{\psi} w_{p}(\underline{x}, \psi) w_{s}(\underline{x}, \psi) L_{t-1}(\underline{x})}{\sum_{\psi} w_{p}(\underline{x}, \psi) w_{s}(\underline{x}, \psi)}
$$

where $L_{0}=u_{0}, t$ is the scaling parameter, $\psi$ defines a local neighborhood, and $w_{p}$ and $w_{s}$ denotes photometric and spatial weights on $\underline{x}$ respectively,

$$
\begin{gathered}
w_{p}(\underline{x}, \psi)=e^{-\frac{1}{2}\left(\frac{\left\|L_{t-1}(\underline{x})-L_{t-1}(\psi)\right\|}{\sigma_{p}}\right)^{2}} \\
w_{s}(\underline{x}, \psi)=e^{-\frac{1}{2}\left(\frac{\|\underline{x}-\psi\|}{\sigma_{s}}\right)^{2}}
\end{gathered}
$$

The key advantage of an iterative bilateral scale space over existing linear and nonlinear scale spaces is that that coarsescale structures are well preserved and localized while fine scale structures such as streaking artifacts and other artifacts that affect region boundary clarity are suppressed. Therefore, by solving the Mumford-Shah energy minimization problem across multiple scales in iterative bilateral scale space, we are able to evolve $\Gamma$ to converge around the contrast intervertebral disc regions while avoiding local minima caused by streaking artifacts and other fine scale artifacts. Based on the above iterative bilateral scale space formulation, the proposed multiscale segmentation method can be described as follows. Let $L$ be the iterative bilateral scale space representation of $u_{o}$ at $k$ scales. At scale $t-1$, an estimate of $R$ is determined by minimizing the Mumford Shah energy functional for $L_{t-1}$ using the functions $u_{1, t}, \ldots, u_{n, t}$ associated with the estimate of $R$ at $t$ as the initial condition,

$\hat{R}_{t-1}=\underset{R_{t-1}}{\arg \min }\left[\alpha \oint_{R}\left(u-L_{t-1}\right)^{2} d \underline{x}+\oint_{R-\Gamma}\|\nabla u\|^{2} d \underline{x}+\beta|\Gamma|\right]$.

This hierarchical estimation process is performed going from the coarsest scale to the finest scale to obtain the final segmented regions $\hat{R}=\hat{R}_{0}$. Based on testing, setting the number of scales to three $(t=\{0,1,2\}$ ) was found to be effective and is used for all tests.

\section{B. Volumetric Reconstruction}

Based on the segmented contrasted intervertebral disc regions obtained during the segmentation process, a set of $q$ points $P=\left\{\underline{p}_{1}, \ldots, \underline{p}_{q}\right\}$ is extracted along the intervertebral disc boundaries to represent points along the surface of the intervertebral disc. A Delaunay triangulation [8] $D(P)$ is then performed based on $P$ to construct the volumetric representation of the intervertebral disc. The Delaunay triangulation, in the case of three-dimensional spaces, is based on the criteria that the circumscribed spheres of all triangles in $D(P)$ must not contain points other than the three points defining the triangle itself. The problem of computing the Delaunay triangulation of $P$ is solved using the Quickhull algorithm [13].

\section{EXPERIMENTAL RESULTS}

To evaluate the effectiveness of the proposed system, intervertebral disc segmentation and volumetric reconstruction was performed on three test sets of serial PQCT images acquired from the porcine functional spinal units at three different times:

1) Test 1: prior to applying flexion/extension motions with compressive load.

2) Test 2: after 7000 cycles of flexion/extension motions with a $1.5 \mathrm{kN}$ compressive load to cause partial damage to the intervertebral disc [7].

3) Test 3: after additional cycles of vibrational motions to exacerbate herniation.

All test sets consists of eight slices cover an entire intervertebral disc and are representative of non-ideal, realworld scenarios, where the PQCT images are characterized by streaking artifacts and unclear separation between contrasted intervertebral disc and surrounding bone regions.

The mean absolute distance (MAD) error between the ground truth measurements by a trained expert and the obtained intervertebral disc regions using the proposed system is computed over all images within the test sets. The MAD error results for all three test sets are shown in Table I. The MAD error of the segmented intervertebral regions produced by the proposed system is close to the ground-truth measurements, with all tests having MAD error of under $0.8 \mathrm{~mm}$. Sample segmentation results from all three tests are shown in Fig. 3. Visually, the proposed system was able to segment the contrasted intervertebral disc regions with a high level of 


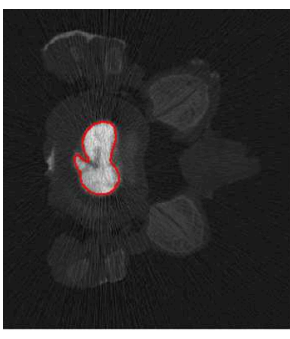

Test 1

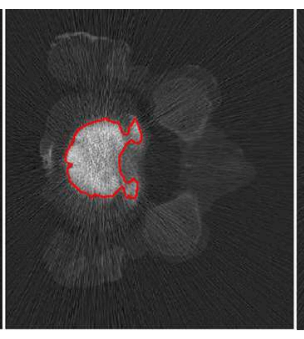

Test 2

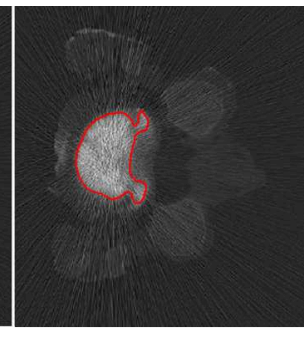

Test 3
Fig. 3. Sample intervertebral disc segmentation results for all three tests Visually, the proposed system was able to segment the contrasted intervertebral disc regions with a high level of accuracy for all tests.
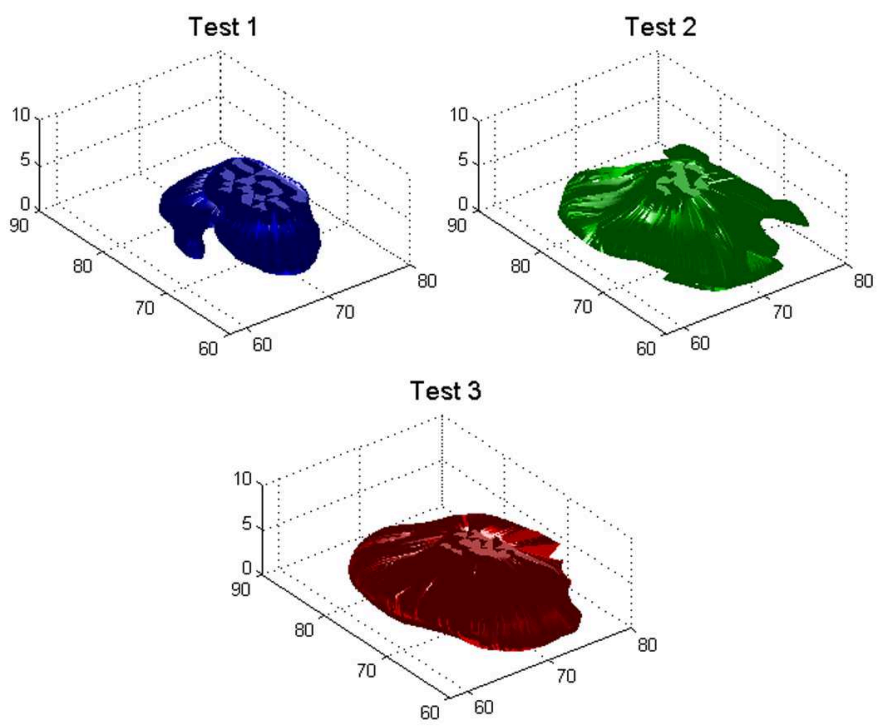

Fig. 4. The volumetric representations of the intervertebral disc for all three tests. Such representations allow the migration of nucleus pulposus in herniated intervertebral discs to be visualized and quantified in a systematic fashion.

accuracy for all tests. This demonstrates the effectiveness of the proposed method in segmenting intervertebral disc regions from PQCT imagery. The volumetric representations of the intervertebral disc for all three tests are shown in Fig. 4. Such representations allow the migration of nucleus pulposus in herniated intervertebral discs to be visualized and quantified in a systematic fashion.

TABLE I

MEAN ABSOLUTE DisTANCE (MAD) ERROR BETWEEN GROUND TRUTH MEASUREMENTS AND INTERVERTEBRAL DISCS GENERATED BY THE PROPOSED SYSTEM.

\begin{tabular}{|c|c|c|}
\hline \hline \multicolumn{3}{|c|}{ MAD (mm) } \\
\hline Test 1 & Test 2 & Test 3 \\
\hline \hline 0.40 & 0.58 & 0.74 \\
\hline \hline
\end{tabular}

\section{COnClusions And Future Work}

In this paper, we have introduced an automatic approach to the problem of intervertebral disc segmentation and volumetric reconstruction from PQCT imagery. A novel multi-scale level set scheme based on a Mumford Shah model in bilateral scale space was introduced for segmenting contrasted intervertebral disc regions from the serial PQCT images, thereby addressing the issues associated with streak artifacts, unclear density separation between contrasted intervertebral disc and surround bone regions, and multiple contrasted regions. A Delaunay triangulation approach was employed for constructing a volumetric representation of the intervertebral disc based on the segmented contrasted regions. Experimental results showed that good segmentation accuracy of intervertebral disc can be achieved from PQCT image sequences when compared to ground-truth measurements. Future work involves extracting detailed volume and shape information about the intervertebral disc under normal conditions and herniated conditions from the extracted 3D volumes for the purpose of quantifying and studying the migration of nucleus pulposus in herniated intervertebral discs under flexion/compressive motions combined with a compressive load to provide a better understanding of low back pain and intervertebral disc disorders.

\section{ACKNOWLEDGMENT}

The authors would like to thank the Natural Sciences and Engineering Research Council (NSERC) of Canada for funding this project, as well as Dr. Stuart McGill. The authors would also like to thank the Vision and Image Processing Group at the University of Waterloo.

\section{REFERENCES}

[1] N. Hadler, "Work disability and musculoskeletal disease", Arthritis and Rheumatism, vol. 29, pp. 1410-1411, 1986.

[2] W. Marras, S. Lavender, S. Leurgans, S. Rajulu, W. Allread, F. Fathallah, and S. Ferguson, "The role of dynamic three-dimensional trunk motion in occupationally-related low back disorders. The effects of workplace factors, trunk position, and trunk motion characteristics on risk of injury", Spine, vol. 18, pp. 617-628, 1993.

[3] R. Norman, R. Wells, P. Neumann, J. Frank, H. Shannon, and M. Kerr, "A comparison of peak vs. cumulative physical work exposure risk factors for the reporting of low back pain in the automotive industry", Clinical Biomechanics, vol. 13, pp. 561-573, 1998.

[4] C. Tampier , J. Drake, J. Callaghan, and S. McGill, "Progressive disc herniation: An investigation of the mechanism using radiologic, histochemical, and microscopic dissection techniques on a porcine model", Spine, vol. 32, pp. 2869-2874, 2007.

[5] S. Gordon, K. Yang, P. Mayer, A. Mace, V. Kish, and E. Radin, "Mechanism of disc rupture: A preliminary report", Spine, vol. 164, pp. 450-456, 1991.

[6] T. Curry, J. Dowdey, and R. Murry, Christensen's Physics of Diagnostic Radiology Thomas S Curry 4th edition. Philadelphia: Lippincott Williams \& Wilkins, 1990.

[7] J. Callaghan and S. McGill, "Intervertebral disc herniation: Studies on a porcine model exposed to highly repetitive flexion/extension motion with compressive force", Clinical Biomechanics, vol. 16, pp. 28-37, 2001.

[8] L. Guibas, D. Knuth, and M. Sharir, "Randomized incremental construction of Delaunay and Voronoi diagrams", Algorithmica, vol. 7, pp. 381413, 1992.

[9] D. Mumford and J. Shah, "Optimal approximation by piecewise smooth functions and associated variational problems", Communications on Pure and Applied Math, vol. 42, pp. 577685, 1989.

[10] S. Osher and J. Sethian, "Fronts propagating with curvature dependent speed: Algorithms based on Hamilton-Jacobi formulations", Journal of Computational Physics, vol. 79, pp. 12-49, 1988.

[11] C. Tomasi and R. Manduchi, "Bilateral filtering for gray and color images", Proceedings of the IEEE International Conference on Computer Vision, Bombay, India, 1998.

[12] T. Lindeberg, "Scale-space theory: A basic tool for analysing structures at different scales", Journal of Applied Statistics, vol. 21, no. 2, pp. 224270, 1994.

[13] C. Barber, D. Dobkin, and H. Huhdanpaa, "The Quickhull Algorithm for Convex Hulls", ACM Transactions on Mathematical Software, vol. 22, no. 4, p. 469-483, 1996. 